\title{
Prise en charge de l'hyperkaliémie aux urgences
}

\section{Management of Hyperkalemia in the Emergency Department}

\author{
L. Lemoine - M. Legrand - G. Potel - P. Rossignol - E. Montassier
}

Reçu le 18 octobre 2018; accepté le 24 novembre 2018

(C) SFMU et Lavoisier SAS 2018

Résumé L'hyperkaliémie est l'un des désordres hydroélectrolytiques les plus fréquemment rencontrés aux urgences. Les étiologies principales sont l'insuffisance rénale aiguë ou chronique, le diabète et l'insuffisance cardiaque. L'hyperkaliémie aiguë peut être une urgence vitale, car elle est potentiellement létale du fait du risque d'arythmie cardiaque. Sa prise en charge aux urgences manque actuellement de recommandations claires en ce qui concerne le seuil d'intervention et les thérapeutiques à utiliser. Les thérapeutiques couramment appliquées sont fondées sur un faible niveau de preuve, et leurs effets secondaires sont mal connus. Des études supplémentaires sont nécessaires pour évaluer l'utilisation de ces traitements et celle de nouveaux traitements potentiellement prometteurs. Nous faisons ici une mise au point sur les données connues en termes d'épidémiologie, de manifestations cliniques et électrocardiographiques, et

\footnotetext{
L. Lemoine

Service des urgences, CHU de Nantes,

F-44000 Nantes, France

M. Legrand

Service de réanimation, hôpital Saint-Louis,

Assistance Publique-Hôpitaux de Paris

avenue Claude Vellefaux,

F-75010 Paris, France

Université Paris Diderot, UMR Inserm S942,

F-75010 Paris, France

M. Legrand $\cdot$ P. Rossignol $\cdot$ E. Montassier $(\square)$

F-CRIN INI-CRCT,

F-54500 Vandœuvre-lès-Nancy, France

e-mail : Emmanuel.MONTASSIER@chu-nantes.fr

G. Potel · E. Montassier

Service des urgences, CHU de Nantes,

F-44000 Nantes, France

Laboratoire MiHAR, université de Nantes,

F-44000, Nantes, France

P. Rossignol

Centre d'investigation clinique CIC-P1433,

Inserm, Nancy, F-54500 Vandœuvre-lès-Nancy, France
}

des différentes thérapeutiques qui peuvent être proposées dans la prise en charge de l'hyperkaliémie aux urgences.

Mots clés Hyperkaliémie · Arythmie cardiaque · Urgence · Insuffisance rénale $\cdot$ Bêta agoniste adrénergique $\cdot$ Insuline

Abstract Hyperkalemia is one of the most common electrolytic disorders in the emergency department. Chronic kidney disease and acute kidney injury, diabetes and heart failure are the main etiologies. Management of hyperkalemia can be a life-saving emergency because of its lethal potential. It can lead to cardiac arrhythmias and cardiac conduction disorders. Its management in the emergency department currently lacks clear guidelines regarding the intervention threshold and the therapeutics used. Standard therapies are based on a low level of evidence, and their side effects are poorly understood. Further studies are needed to evaluate the use of these treatments and potentially promising new treatments. In this article, we focus on known data in terms of epidemiology, clinical and electrographic manifestations, and the different medications that can be proposed in the management of hyperkalemia in the emergency department.

Keywords Severe hyperkalemia $\cdot$ Arrhythmias - Cardiac · Emergency treatment $\cdot$ Renal insufficiency $\cdot$ Adrenergic beta-agonists $\cdot$ Insulin

\section{Introduction}

La kaliémie est normalement comprise entre d'étroites limites, classiquement 3,5 à $5 \mathrm{mmol} / \mathrm{l}$, et maintenue par de multiples mécanismes, rénaux et extrarénaux, constituant l'homéostasie du potassium. Globalement, $98 \%$ du potassium est réparti à l'intérieur de la cellule et seulement $2 \%$ à l'extérieur. Cette asymétrie, maintenue par la pompe $\mathrm{Na}^{+}-\mathrm{K}^{+}$ ATPase, est essentielle à l'équilibre du potentiel de repos de la membrane cellulaire et à la diffusion du potentiel d'action. Cela contribue au bon fonctionnement de la conduction 
nerveuse et de la contraction musculaire, et tout spécialement du tissu cardiaque $[1,2]$.

L'hyperkaliémie est un trouble électrolytique fréquent parmi les patients admis aux urgences [3]. Globalement, l'incidence de l'hyperkaliémie se situe entre 1 et $10 \%$ des patients hospitalisés [4]. En France, aux urgences, des données non publiées, obtenues auprès de plusieurs services d'urgences, montrent une incidence entre 1,5 et 2,7 \% des admissions. L'hyperkaliémie est potentiellement grave du fait du risque de troubles du rythme ventriculaire. Plusieurs études ont démontré l'association entre hyperkaliémie aiguë et mortalité [5-7]. L'hyperkaliémie est habituellement classée en légère, modérée ou sévère $[3,8,9]$; cependant, les seuils pour définir la gravité de l'hyperkaliémie sont inconstants.

L'objectif de notre mise au point est de rapporter les données les plus récentes concernant les étiologies, les manifestations cliniques et électrocardiographiques, ainsi que les différentes thérapeutiques qui peuvent être proposées dans la prise en charge de l'hyperkaliémie aux urgences.

\section{Hyperkaliémie aiguë : principales étiologies}

Il est important en premier lieu d'éliminer des causes de pseudohyperkaliémie, qui sont : hyperleucocytose supérieure à $70 \mathrm{G} / 1$, thrombocytose supérieure à $600 \mathrm{G} / 1$, pose prolongée du garrot, hémolyse [2].

Il est bien établi que la principale cause d'hyperkaliémie est l'insuffisance rénale, qu'elle soit aiguë ou chronique, les autres étiologies étant le diabète et l'insuffisance cardiaque, d'autres mécanismes peuvent être en cause : nécrose tissulaire, acidose métabolique, rhabdomyolyse [10,11]. Les thérapeutiques utilisées dans cette pathologie, interférant dans l'homéostasie du potassium, précipitent l'élévation de la kaliémie. Ainsi, une iatrogénie est fréquemment impliquée dans les causes de l'hyperkaliémie aiguë, en lien avec une insuffisance rénale aiguë [10].

Dans le syndrome cardiorénal, la dysfonction cardiaque et celle rénale peuvent coexister. L'insuffisance cardiaque congestive, la diminution du débit de filtration rénale et l'hypervolémie s'intriquent, pouvant conduire à la survenue d'une hyperkaliémie modérée à sévère [12].

\section{Hyperkaliémie aiguë : manifestations cliniques et électrocardiographiques}

Les patients en hyperkaliémie peuvent ne présenter aucun symptôme ou des symptômes aspécifiques comme une faiblesse, une asthénie, des paresthésies, des crampes ou une dyspnée pouvant aller jusqu'à la détresse respiratoire [9]. Il est important de noter que ces manifestations cliniques ne sont aucunement prédictives de la gravité ou de la sévérité d'une hyperkaliémie aiguë.

En ce qui concerne l'électrocardiogramme, l'hyperkaliémie modérée est souvent associée à une accélération de la repolarisation, des changements de l'onde $\mathrm{T}$ en résultent directement [13] (Fig. 1). Des ondes T pointues sont considérées comme le signe le plus précoce d'hyperkaliémie [14]. Une hyperkaliémie légère à modérée est responsable d'une diminution de la conduction électrique entre les myocytes cardiaques, ce qui provoque un allongement progressif de l'espace PR et un élargissement progressif des QRS alors que le taux de potassium augmente [9]. Les myocytes atriaux sont plus sensibles à l'hyperkaliémie. En résulte une diminution de l'amplitude de l'onde P évoluant progressivement vers un aplatissement de l'onde $\mathrm{P}$, sans extinction du nœud sinoatrial. Certains rapportent que les impulsions du nœud sinoatrial sont directement transmises au nœud atrioventriculaire sans dépolariser l'oreillette. Plus l'hyperkaliémie augmente et plus les conductions sinoatriale et atrioventriculaire diminuent, pouvant aboutir à un échappement ventriculaire [14]. Ensuite, les QRS continuent de s'élargir jusqu'à se mélanger à l'onde T. À l'extrême, une hyperkaliémie sévère peut entraîner un passage en fibrillation ventriculaire ou en asystolie [14]. D'autres anomalies associées à l'hyperkaliémie ont été décrites dans la littérature : un sous-décalage du segment ST, un hémibloc, un bloc de branche droit ou gauche, un bloc bifasciculaire ou trifasciculaire. Il peut même apparaître des signes d'infarctus du myocarde, un sus-décalage diffus du segment ST ou dans les dérivations inférieures [14]. On n'observe généralement pas d'ondes Q. Cependant, il est important de noter que la relation entre taux de potassium et apparition de modifications électrocardiographiques est inconstante, la sensibilité de l'ECG isolé pour détecter une hyperkaliémie étant seulement de 34 à $43 \%[15,16]$. Ainsi, un ECG normal peut être obtenu même avec une hyperkaliémie sévère, d'autant plus que l'hyperkaliémie s'est installée lentement [13,17]. Au total, les conséquences cardiaques de l'hyperkaliémie sont essentiellement liées à sa rapidité d'installation et au terrain sous-jacent $[18,19]$.

\section{Hyperkaliémie aiguë : prise en charge thérapeutique}

Il n'existe actuellement pas de stratégie thérapeutique fondée sur un haut niveau de preuve dans le traitement urgent de l'hyperkaliémie aiguë. Cela est principalement dû au peu d'essais randomisés réalisés et à l'hétérogénéité du seuil défini pour débuter un traitement entre les différentes études. Des recommandations claires manquent concernant le seuil d'hyperkaliémie pour l'indication d'un traitement en urgence, et le choix du traitement, qui n'a pas changé au 


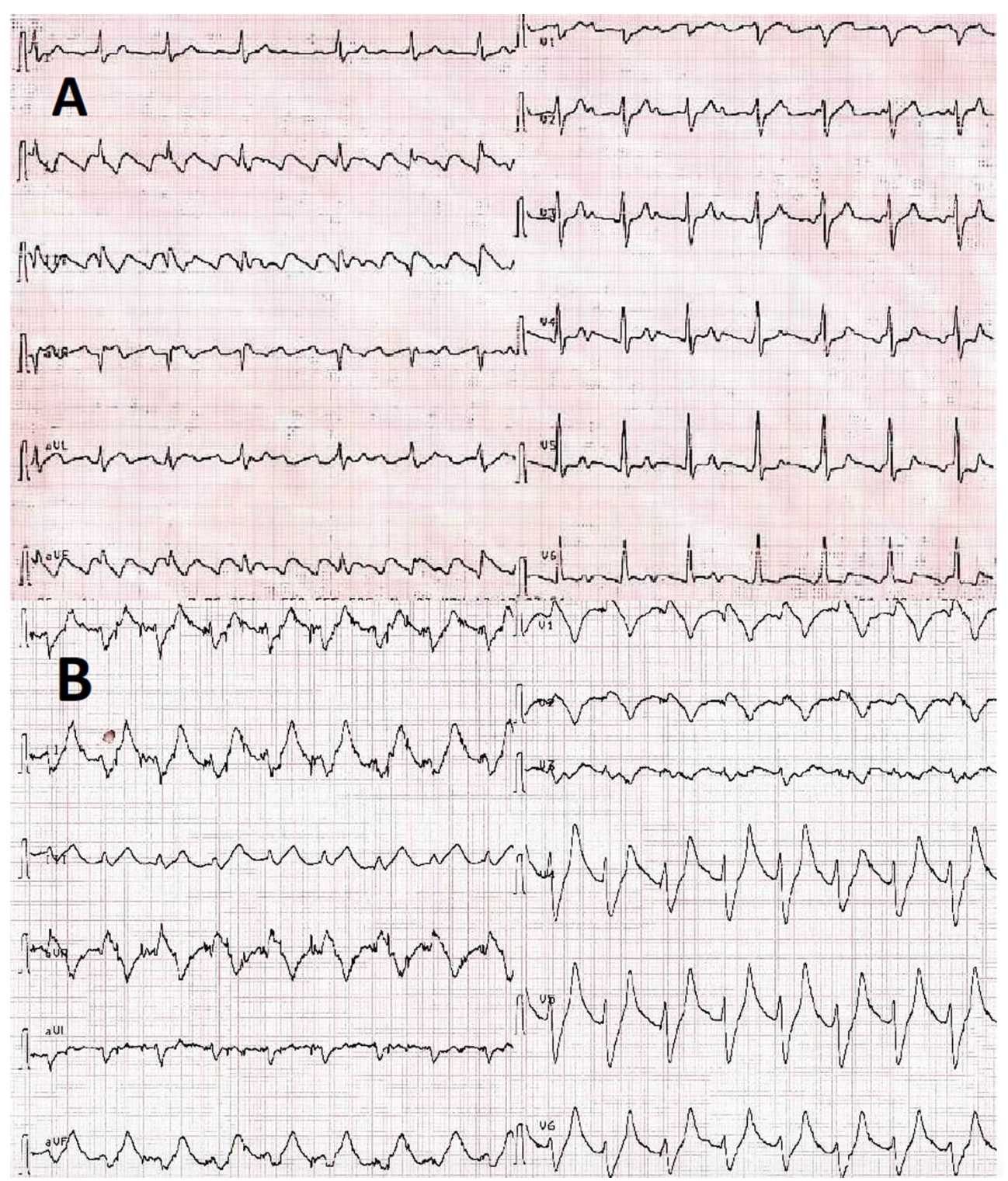

Fig. 1 Exemples de modifications électrocardiographiques en rapport avec une hyperkaliémie. A. Élargissement de l'espace PR. B. Ondes T amples et pointues et élargissement des QRS

cours des dernières années, est bien souvent subjectif $[3,4]$. Il est habituellement admis qu'un traitement doit être débuté en urgence lorsque le taux de potassium est supérieur à $6 \mathrm{mmol} / \mathrm{l}$ et/ou lorsque l'on retrouve des signes électrocardiographiques d'hyperkaliémie à l'ECG $[3,20]$. Dans la plupart des cas, plus d'un agent est utilisé. Plusieurs revues ont synthétisé les différentes options thérapeutiques [20-23].

La prise en charge initiale de l'hyperkaliémie aiguë aux urgences après confirmation de l'absence de pseudohyperkaliémie se divise en trois étapes :

- d'abord l'antagonisation de l'effet de l'hyperkaliémie sur la potentielle hyperexcitabilité myocardique afin de protéger des troubles du rythme et de la conduction;
- puis la diminution de la kaliémie en elle-même, en faisant passer le potassium du secteur extracellulaire vers le secteur intracellulaire ;

- enfin, l'élimination du potassium, que cela soit par le biais d'une épuration digestive (résine échangeuse d'ions), rénale (traitement diurétique) ou extrarénale (hémodialyse).

\section{Stabiliser la membrane cardiaque}

Même si aucune étude ne démontre l'intérêt de l'administration d'un sel de calcium dans le traitement de l'hyperkaliémie, cette prescription est largement recommandée $[4,23]$. 
Le calcium permet de stabiliser la membrane cardiaque en diminuant le risque d'arythmie causée par l'hyperkaliémie. Il n'a donc aucun rôle dans la diminution de la kaliémie. L'administration intraveineuse d'un sel de calcium (chlorure de calcium ou gluconate de calcium) est recommandée dès l'apparition d'anomalies électriques en rapport avec l'hyperkaliémie à l'ECG (ondes T pointues). Le chlorure de calcium contient plus de calcium que le gluconate, mais présente un risque de nécrose tissulaire en cas d'extravasation. Ainsi, on préférera l'utilisation du gluconate de calcium (à $10 \%$, $10 \mathrm{ml}$ sur dix min, soit 2,25 mmol de calcium). Sa rapidité d'action est quasi immédiate, et sa durée d'action de 30 à $60 \mathrm{~min}$. Il est possible de répéter la dose de gluconate de calcium si l'ECG ne se normalise pas dans les cinq min ou si les anomalies réapparaissent après amélioration [13]. L'utilisation d'un sel de calcium n'est cependant pas recommandée en cas d'hypercalcémie ou de surdosage ou intoxication en digitaliques.

\section{Faire rentrer du potassium dans les cellules}

\section{$2 \beta$ mimétiques}

L'utilisation des $\beta 2$ mimétiques a été largement étudiée dans le traitement à la phase aiguë de l'hyperkaliémie $[20,23]$. En stimulant la pompe Na-K ATP, le potassium est réabsorbé à l'intérieur de la cellule. L'efficacité maximale du salbutamol sur la diminution du potassium est observée entre 30 et $60 \mathrm{~min}$ et dure environ deux heures. Les résultats des différentes études sont présentés dans l'Annexe A [24-29]. En résumé, avec l'utilisation des $\beta 2$ mimétiques, la diminution de la kaliémie est d'environ $1 \mathrm{mmol} / 1$ en $60 \mathrm{~min}$, avec une efficacité similaire entre une utilisation intraveineuse (à la dose de $0,5 \mathrm{mg}$ sur $15 \mathrm{~min}$ ) ou en nébulisation (meilleure efficacité à une dose de $20 \mathrm{mg}$ qu'à $10 \mathrm{mg}$ ) [20].

Parmi les effets secondaires rapportés, on peut observer une tachycardie, des tremblements, une nervosité, des palpitations. À noter que les patients sous $\beta$-bloquants étaient exclus dans plusieurs études évaluant l'efficacité des $\beta 2$ mimétiques, les auteurs justifiant cette exclusion par un effet moindre attendu sur l'hyperkaliémie dans cette population, sans que toutefois cela ait pu être corroboré par d'autres études $[9,13]$. De plus, deux études incluant des patients hémodialysés rapportent qu'environ $40 \%$ des patients présentaient une résistance aux $\beta 2$ mimétiques, avec une diminution moindre de la kaliémie chez ces patients, sans pouvoir prédire quelle sera la réponse au traitement. Le mécanisme n'est pas connu ; cependant, cette résistance $\beta 2$ mimétiques n'a été rapportée à ce jour que chez des patients déjà en hémodialyse $[24,25]$. Cependant, certains auteurs proposent que le salbutamol ne soit pas utilisé seul [13].

\section{Insulinothérapie}

L'association insuline-glucose diminue la kaliémie en réabsorbant le potassium à l'intérieur de la cellule par l'activation de la pompe Na-K ATPase. Cet effet est indépendant de l'effet hypoglycémiant de l'insuline. L'injection de glucose seul n'est pas recommandée, car le taux d'insuline endogène libéré sera probablement insuffisant, et le risque d'exacerbation de l'hyperkaliémie est important du fait de l'effet hypertonique entrâné par un transfert d'eau et de potassium en dehors de la cellule secondaire à l'hyperglycémie.

Plusieurs stratégies de traitement ont été étudiées et sont rapportées dans l'Annexe B [25,26,30-36]. En résumé, une injection de dix unités d'insuline rapide en bolus suivie par un bolus de $25 \mathrm{~g}$ de glucose permet de faire baisser la kaliémie de 0,6 à $1 \mathrm{mmol} / \mathrm{l}$ dès $15 \mathrm{~min}$, avec un pic d'efficacité entre 30 et $60 \mathrm{~min}$ et une durée d'action de quatre à six heures [25]. Le choix de la dose d'insuline (10 à 20 unités) n'était pas associé à une différence significative sur la diminution de la kaliémie [37]. L'administration d'une dose inférieure d'insuline a aussi été évaluée [38,39]. À noter que dans une étude testant l'insuline administrée en perfusion continue sur quatre heures, la kaliémie réaugmentait à $90 \mathrm{~min}$ [23]. Cette stratégie de perfusion continue n'est donc pas recommandée.

Parmi les effets secondaires décrits, le principal est le risque d'hypoglycémie [37,40]. Celui-ci est fréquent et probablement sous-estimé [40]. L'hypoglycémie étant souvent retardée (une à trois heures après l'injection) du fait d'un rebond initial de la glycémie après l'injection, mais aussi parce que l'utilisation de glucose est souvent trop faible par rapport à l'apport exogène d'insuline. De plus, la demivie de l'insuline est prolongée s'il existe une insuffisance rénale, l'apport de glucose doit alors être prolongé [41]. Dans une revue incluant 11 études, environ $20 \%$ des patients présentait une hypoglycémie [37]. Cependant, dans une autre étude, aucun patient ne présentait d'hypoglycémie après $60 \mathrm{~g}$ de glucose associés à 20 unités d'insuline (sur une heure en IVSE) [26].

Ainsi, une approche proposée est l'administration d'un bolus de six unités d'insuline rapide, suivi par une perfusion de 20 unités d'insuline sur une heure associée à $60 \mathrm{~g}$ de glucose (soit $600 \mathrm{ml}$ de glucose $10 \%$ ) sur une heure [41].

\section{Association $\beta 2$ mimétiques et insulinothérapie}

Plusieurs stratégies de traitement associant $\beta 2$ mimétiques et insulinothérapie ont été étudiées et sont rapportées dans l'Annexe $C[25,31,35]$. En résumé, l'association $\beta 2$ mimétiques $(20 \mathrm{mg}$ de salbutamol nébulisé) et insuline-glucose (dix unités d'insuline rapide sur une heure et $50 \mathrm{ml}$ de solution à $50 \%$ de glucose) permet de faire baisser d'environ $1,2 \mathrm{mmol} / 1$ la kaliémie, $60 \mathrm{~min}$ après l'administration des 
deux thérapeutiques. Cette diminution de la kaliémie était significative par rapport aux deux traitements utilisés séparément. La différence sur la baisse de la kaliémie était significative dès $15 \mathrm{~min}$ et jusqu'à $60 \mathrm{~min}$.

En outre, l'ajout d'un $\beta 2$ mimétique à l'insuline diminuait le risque d'hypoglycémie. À l'inverse, l'augmentation de la fréquence cardiaque était significativement supérieure. Nous proposons de réserver cette association aux hyperkaliémies supérieures ou égales à $6,5 \mathrm{mmol} / \mathrm{l}$ ou en cas d'hyperkaliémie avec modifications électrocardiographiques.

\section{Bicarbonates}

Les données sur l'utilisation du bicarbonate de sodium sont contradictoires [21,42]. Ainsi, les patients qui présentaient une baisse du taux de potassium après l'administration de bicarbonate de sodium étaient en acidose métabolique majeure. Une autre étude n'a pas montré d'effet rapide sur la kaliémie après injection de bicarbonate de sodium [31]. En outre, cette thérapeutique n'est pas dénuée d'effets secondaires, principalement hypernatrémie et alcalose métabolique. Ces effets secondaires seraient potentiellement sévères dans la population de patients présentant le plus souvent une hyperkaliémie aux urgences, à savoir les insuffisants cardiaques qui pourraient alors présenter un œdème aigu pulmonaire au décours de la prise en charge de l'hyperkaliémie [9].

Ainsi, nous proposons que l'administration de bicarbonate ne soit pas considérée comme un traitement aux urgences de l'hyperkaliémie, en particulier du fait du risque d'œdème pulmonaire cardiogénique et d'une diminution souvent modeste de la kaliémie. Une indication de ce traitement peut cependant être retenue : chez des patients en hypovolémie majeure et en acidose métabolique [41].

\section{Faire sortir le potassium de l'organisme}

\section{Résines échangeuses de cations}

Les résines échangeuses de cations fonctionnent en échangeant le sodium qu'elles contiennent avec le potassium excrété dans le tube digestif, majoritairement dans le côlon [13]. Néanmoins, l'efficacité est hautement variable, retardée et imprévisible. Ainsi, les études revues retrouvaient une diminution de $0,4 \mathrm{mmol} / \mathrm{l}$ entre 12 et 24 heures de la kaliémie, mais décrivaient aussi la survenue d'hypokaliémies [43]. De plus, une étude rétrospective en 2015, retrouvant une diminution significative relative de $0,14 \mathrm{mmol} / 1 \mathrm{de}$ la kaliémie après traitement par résine, considérait ce traitement comme inapproprié du fait de son coût, des potentiels effets secondaires et du faible intérêt clinique [44].

Ainsi, nous proposons que ce traitement ne soit pas considéré comme un traitement d'urgence du fait de son long délai d'action (au moins deux heures et jusqu'à six heures ou plus) et du fait du risque de nécrose intestinale signalé après l'utilisation du kayexalate dans plusieurs cas cliniques $[21,43]$.

\section{Épuration extrarénale}

Une dialyse urgente devrait être considérée chez des patients anuriques résistant aux traitements de première ligne et présentant des troubles électriques sur l'ECG persistant après traitement. Cela est d'autant plus vrai chez des patients en insuffisance rénale aiguë $[3,10]$. Cependant, le délai de mise en route d'une dialyse en fait un traitement de seconde ligne, la priorité étant toujours pour le médecin urgentiste d'antagoniser l'effet de l'hyperkaliémie sur la potentielle hyperexcitabilité myocardique et de faire passer le potassium du secteur extracellulaire vers le secteur intracellulaire. Ainsi, aux urgences, les traitements précédemment proposés ( $2 \beta$ mimétiques et insuline-glucose) restent à faire en première intention, avant de discuter une éventuelle procédure de dialyse.

\section{Synthèse et perspectives}

L'hyperkaliémie est un trouble électrolytique fréquent aux urgences. Du fait des conséquences potentiellement délétères de l'hyperkaliémie et notamment cardiaques, sa prise en charge est une urgence médicale. L'hétérogénéité des avis d'experts en ce qui concerne le seuil d'intervention et les thérapeutiques à utiliser est importante. Des études évaluent les options thérapeutiques couramment utilisées, mais le niveau de preuve est limité par des essais portant sur de petites cohortes de patients, et l'application des différents traitements est bien souvent soutenue par la subjectivité du prescripteur. Cependant, il est indispensable de débuter rapidement une injection de sels de calcium en cas d'anomalie électrocardiographique, celle-ci n'ayant pas d'effet sur la kaliémie, mais un effet protecteur cardiaque.

En ce qui concerne la prise en charge initiale de l'hyperkaliémie aux urgences, le traitement peut débuter pour une kaliémie supérieure ou égale à $6 \mathrm{mmol} / \mathrm{l}$ et/ou en cas d'anomalie électrocardiographique. Le traitement pourra reposer sur l'insuline-glucose et/ou les $\beta 2$ mimétiques, mais nous proposons de ne pas retenir la prescription de bicarbonates aux urgences du fait de ses effets secondaires potentiels. Les $\beta 2$ mimétiques, du fait de leur simplicité d'utilisation en nébulisation, doivent trouver toute leur place aux urgences. Il est important de rappeler que l'insuline-glucose est pourvoyeuse d'hypoglycémie ou d'hyperglycémie, et que par conséquent la glycémie doit être surveillée au cours de la prise en charge. Ces hypoglycémies peuvent être retardées notamment en cas d'insuffisance rénale. Enfin, au vu du délai maximal d'action des différentes stratégies 

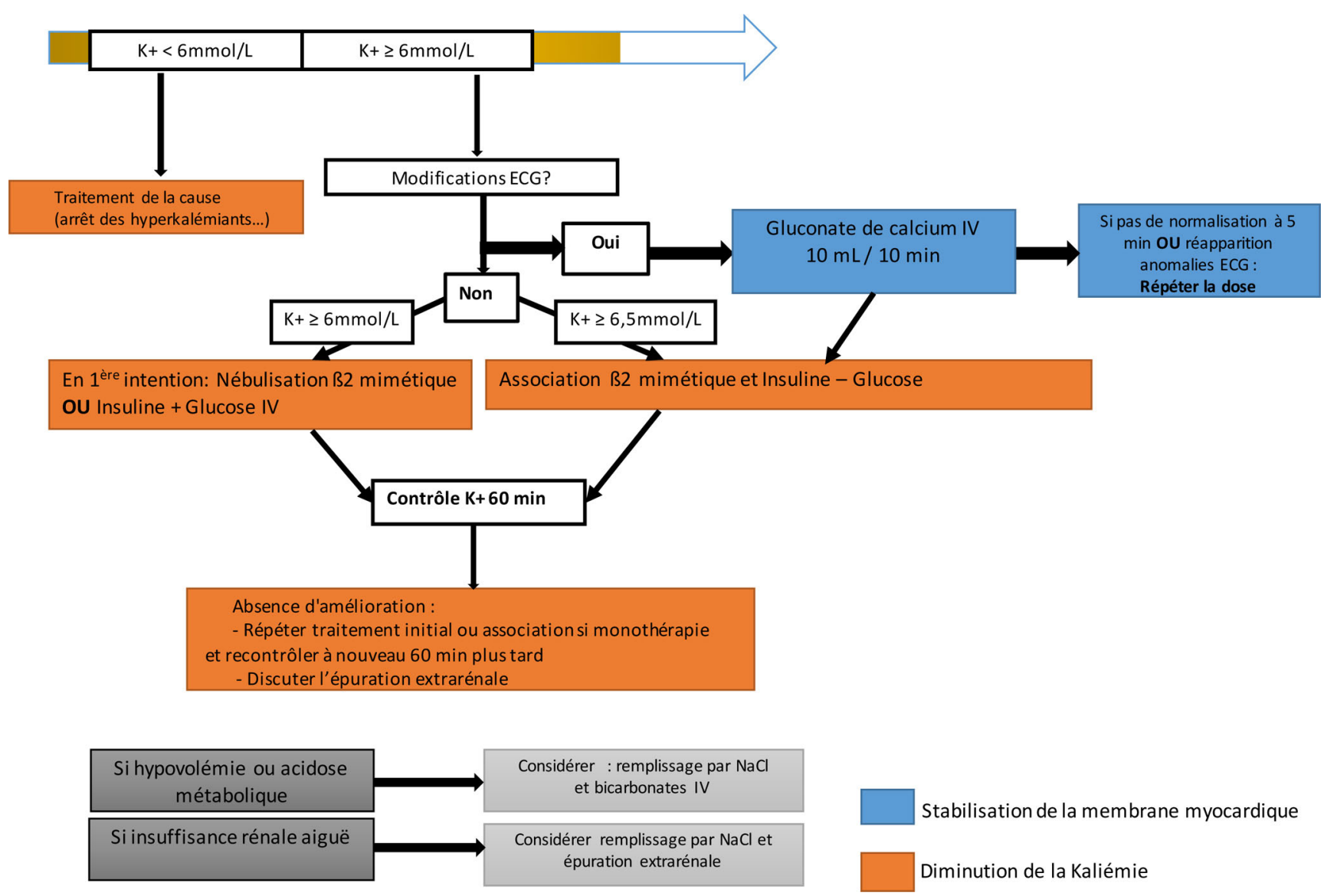

Stabilisation de la membrane myocardique Diminution de la Kaliémie

Fig. 2 Algorithme de prise en charge initiale de l'hyperkaliémie aux urgences. K+ : kaliémie

thérapeutiques, il est proposé un contrôle de la kaliémie après une heure. Les propositions thérapeutiques pour la prise en charge initiale aux urgences sont synthétisées dans la figure 2.

Dans ce contexte, l'importance de définir le niveau de kaliémie auquel le risque de décès ou d'arythmie augmente de manière significative est une priorité, afin de définir à quel taux débuter un traitement agressif. Des données claires manquent également sur la rapidité du traitement nécessaire, alors qu'il est important de connaître quels patients doivent être traités rapidement, et ceux pour lesquels la kaliémie peut être diminuée progressivement. Des études observationnelles sont en cours, mais des essais randomisés devront être menés afin d'évaluer les traitements de première ligne de l'hyperkaliémie aux urgences [45].

Une autre piste de recherche concerne aussi les nouveaux traitements potentiels de l'hyperkaliémie [46]. En effet, le patiromer et le ZS-9 présentent une efficacité potentielle dans des études préliminaires et devront être testés afin de déterminer leur effet prévisible sur la diminution de la kaliémie à la phase initiale et leur rapidité d'action en comparaison avec des traitements standard.

\section{Conclusion}

L'hyperkaliémie est un trouble électrolytique fréquent aux urgences et est potentiellement grave par trouble du rythme cardiaque. Sa prise en charge initiale doit donc être agressive, même si le seuil de kaliémie pour intervention et les thérapeutiques à appliquer sont hétérogènes. Nous avons proposé ici une mise au point sur stratégie de prise en charge initiale de l'hyperkaliémie. Des essais randomisés doivent maintenant permettre de renforcer les avis d'experts pour définir une prise en charge optimisée de l'hyperkaliémie aux urgences.

Conflit d'intérêt : les auteurs déclarent ne pas avoir de lien d'intérêt. 


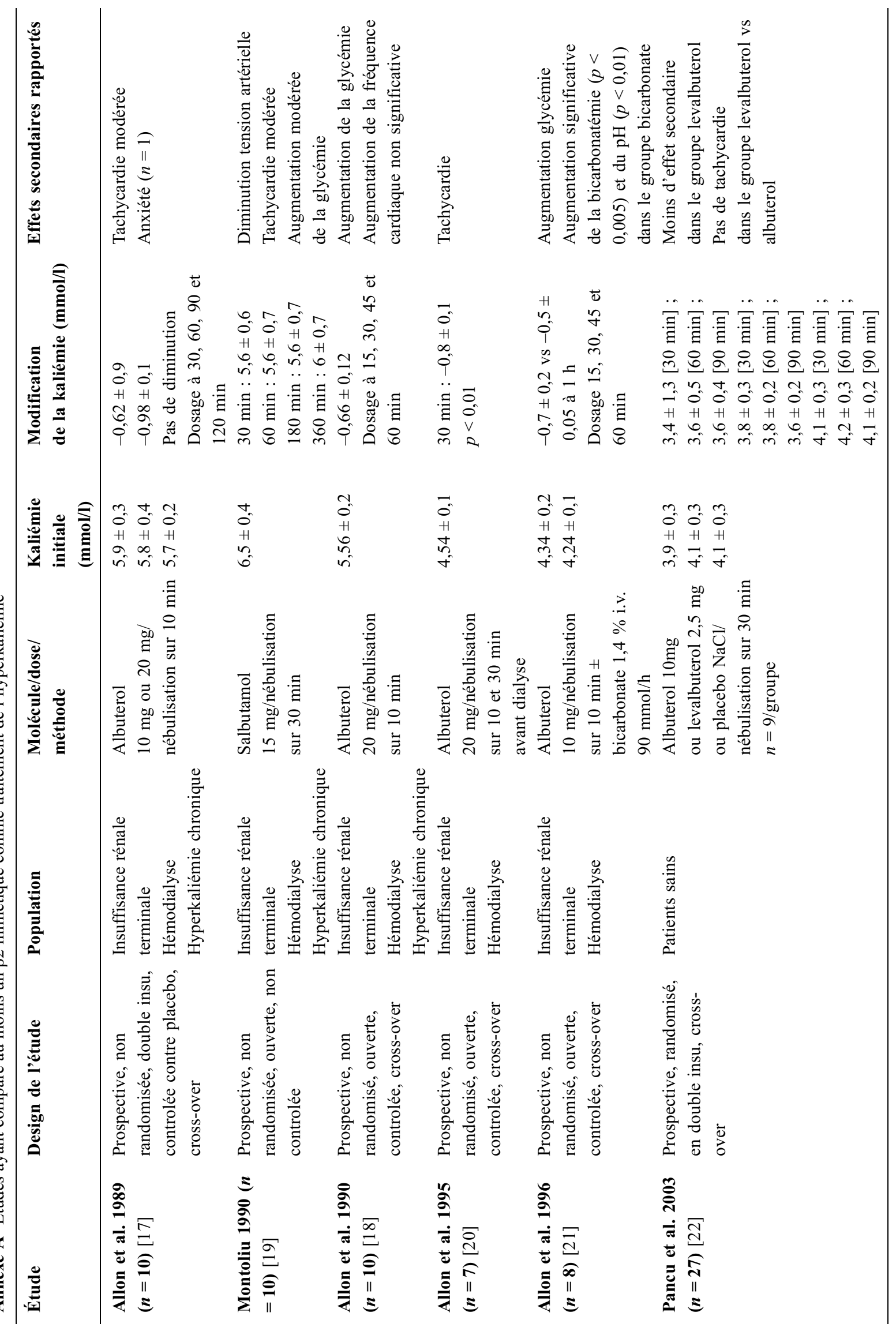

Lavoisier 


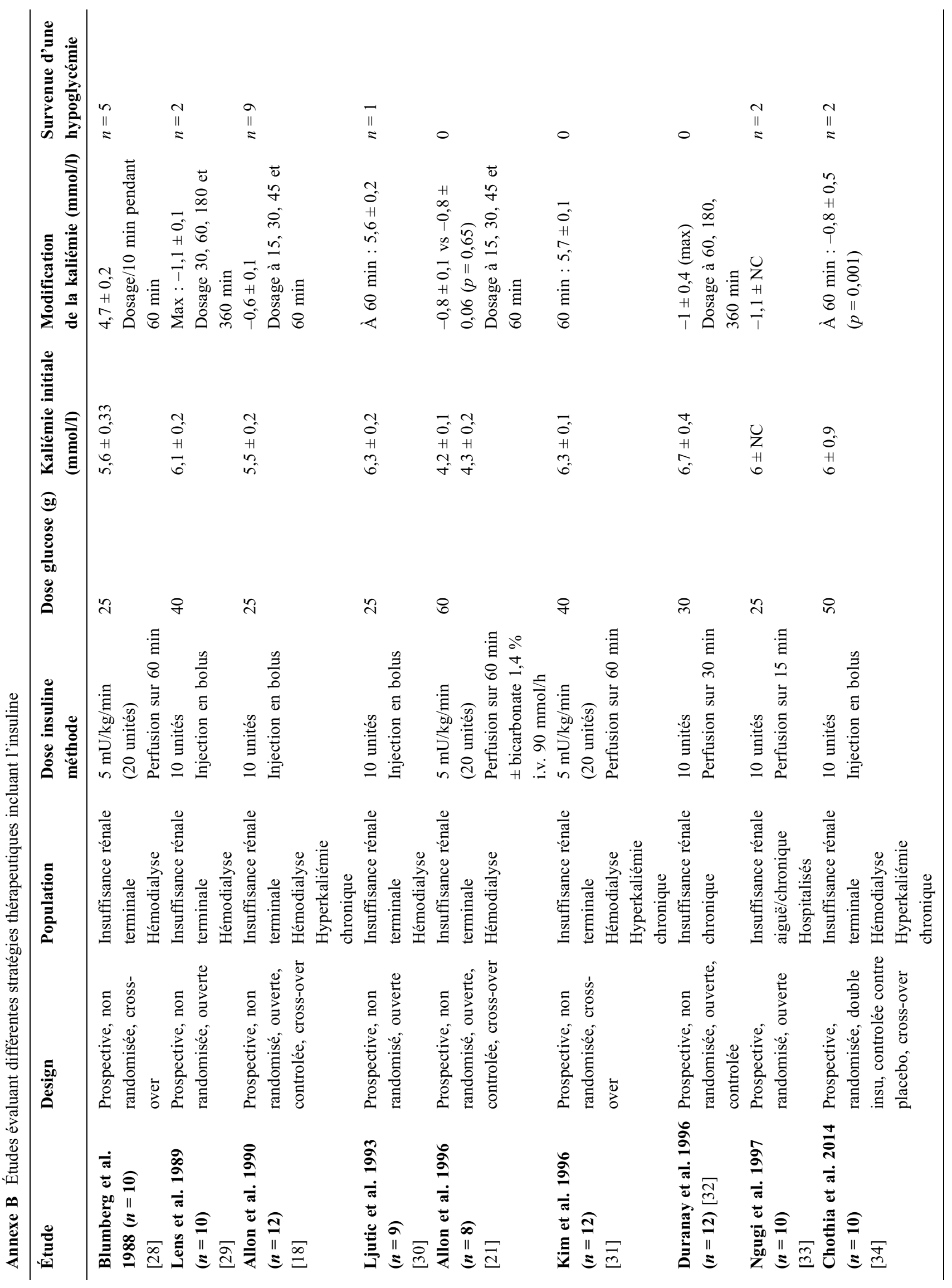




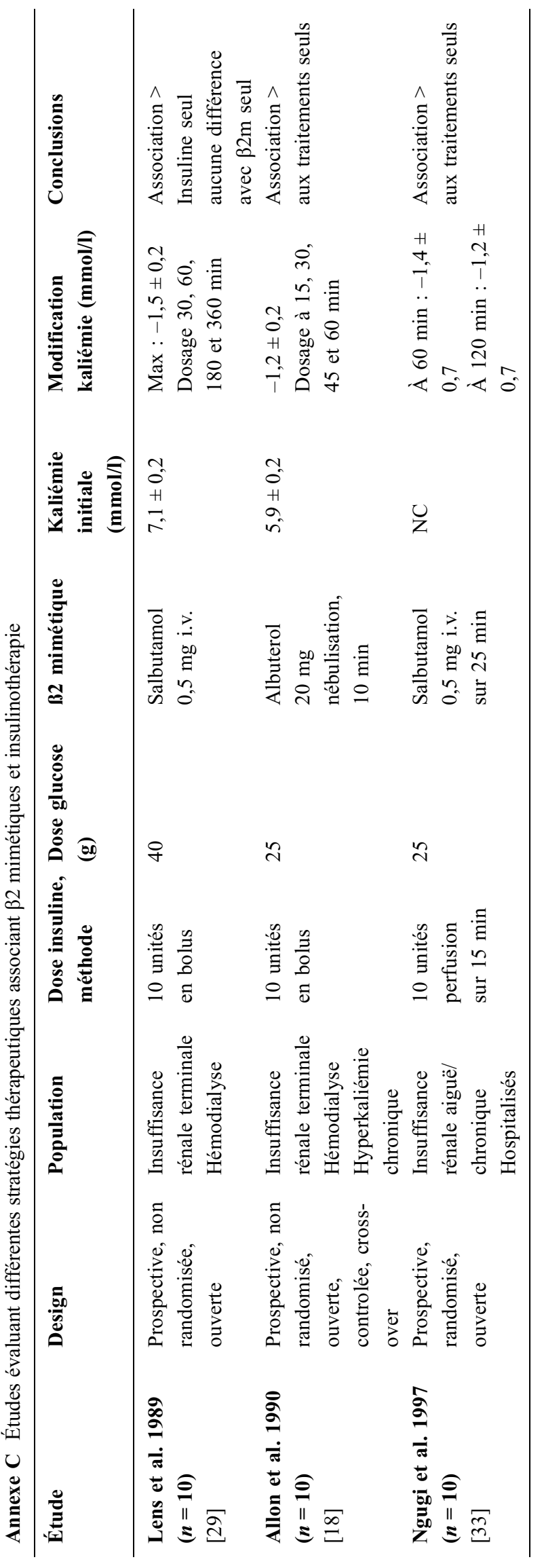

\section{Références}

1. Gumz ML, Rabinowitz L, Wingo CS (2015) An integrated view of potassium homeostasis. N Engl J Med 373:60-72

2. Weiner ID, Linas SL, Wingo CS (2010) Chapter 9 - Disorders of potassium metabolism. In: Floege J, Johnson RJ, Feehally J (eds) Compr Clin Nephrol, Fourth Ed. Mosby, Philadelphia, pp 118-29

3. Rossignol P, Legrand M, Kosiborod M, et al (2016) Emergency management of severe hyperkalemia: guideline for best practice and opportunities for the future. Pharmacol Res 113:585-91

4. Sood MM, Sood AR, Richardson R (2007) Emergency management and commonly encountered outpatient scenarios in patients with hyperkalemia. Mayo Clin Proc 82:1553-61

5. Einhorn LM, Zhan M, Hsu VD, et al (2009) The frequency of hyperkalemia and its significance in chronic kidney disease. Arch Intern Med 169:1156-62

6. McMahon GM, Mendu ML, Gibbons FK, et al (2012) Association between hyperkalemia at critical care initiation and mortality. Intensive Care Med 38:1834-42

7. Jain N, Kotla S, Little BB, et al (2012) Predictors of hyperkalemia and death in patients with cardiac and renal disease. Am J Cardiol 109:1510-13

8. Pepin J, Shields C (2012) Advances in diagnosis and management of hypokalemic and hyperkalemic emergencies. Emerg Med Pract 14:1-17

9. Alfonzo AVM, Isles C, Geddes C, et al (2006) Potassium disorders - clinical spectrum and emergency management. Resuscitation 70:10-25

10. Faubel S, Shah PB (2016) Immediate consequences of acute kidney injury: the impact of traditional and nontraditional complications on mortality in acute kidney injury. Adv Chronic Kidney Dis 23:179-85

11. Muschart X, Boulouffe C, Jamart J, et al (2014) A determination of the current causes of hyperkalaemia and whether they have changed over the past 25 years. Acta Clin Belg 69:280-4

12. Thind GS, Loehrke M, Wilt JL (2018) Acute cardiorenal syndrome: mechanisms and clinical implications. Cleve Clin J Med $85: 231-9$

13. Weisberg LS (2008) Management of severe hyperkalemia: Crit Care Med 36:3246-51

14. Mattu A, Brady WJ, Robinson DA (2000) Electrocardiographic manifestations of hyperkalemia. Am J Emerg Med 18:721-9

15. Wrenn KD, Slovis CM, Slovis BS (1991) The ability of physicians to predict hyperkalemia from the ECG. Ann Emerg Med 20:1229-32

16. Acker CG, Johnson JP, Palevsky PM, et al (1998) Hyperkalemia in hospitalized patients: causes, adequacy of treatment, and results of an attempt to improve physician compliance with published therapy guidelines. Arch Intern Med 158:917-24

17. Szerlip HM, Weiss J, Singer I (1986) Profound hyperkalemia without electrocardiographic manifestations. Am J Kidney Dis $7: 461-5$

18. Montague BT, Ouellette JR, Buller GK (2008) Retrospective review of the frequency of ECG changes in hyperkalemia. Clin J Am Soc Nephrol 3:324-30

19. Surawicz B, Chlebus H, Mazzoleni A (1967) Hemodynamic and electrocardiographic effects of hyperpotassemia. Differences in response to slow and rapid increases in concentration of plasma K. Am Heart J 73:647-64

20. Elliott MJ, Ronksley PE, Clase CM, et al (2010) Management of patients with acute hyperkalemia. CMAJ 182:1631-5

21. Mahoney BA, Smith WA, Lo DS, et al (2005) Emergency interventions for hyperkalaemia. Cochrane Database Syst Rev 2: CD003235. 
22. Kovesdy CP (2015) Management of hyperkalemia: an update for the internist. Am J Med 128:1281-7

23. Batterink J, Cessford TA, Taylor RA (2015) Pharmacological interventions for the acute management of hyperkalaemia in adults. Cochrane Database Syst Rev 10:CD010344

24. Allon M, Dunlay R, Copkney C (1989) Nebulized albuterol for acute hyperkalemia in patients on hemodialysis. Ann Intern Med 110:426-9

25. Allon M, Copkney C (1990) Albuterol and insulin for treatment of hyperkalemia in hemodialysis patients. Kidney Int 38:869-72

26. Allon M, Shanklin N (1996) Effect of bicarbonate administration on plasma potassium in dialysis patients: Interactions with insulin and albuterol. Am J Kidney Dis 28:508-14

27. Allon M, Shanklin N (1995) Effect of albuterol treatment on subsequent dialytic potassium removal. Am J Kidney Dis 26:607-13

28. Montoliu J, Almirall J, Ponz E, et al (1990) Treatment of hyperkalaemia in renal failure with salbutamol inhalation. J Intern Med 228:35-7

29. Pancu D, LaFlamme M, Evans E, et al (2003) Levalbuterol is as effective as racemic albuterol in lowering serum potassium. J Emerg Med 25:13-6

30. Blumberg A, Weidmann P, Shaw S, et al (1988) Effect of various therapeutic approaches on plasma potassium and major regulating factors in terminal renal failure. Am J Med 85:507-12

31. Lens XM, Montoliu J, Cases A, et al (1989) Treatment of hyperkalaemia in renal failure: salbutamol v. insulin. Nephrol Dial Transplant 4:228-32

32. Ljutić D, Rumboldt Z (1993) Should glucose be administered before, with, or after insulin, in the management of hyperkalemia? Ren Fail 15:73-6

33. Kim HJ (1996) Combined effect of bicarbonate and insulin with glucose in acute therapy of hyperkalemia in end-stage renal disease patients. Nephron 72:476-82

34. Duranay M, Ateș K, Ertürk S, et al (1996) Comparison of aminophylline and insulin infusions in treatment of hyperkalemia in patients with end-stage renal disease. Nephron 73:105
35. Ngugi NN, McLigeyo SO, Kayima JK (1997) Treatment of hyperkalaemia by altering the transcellular gradient in patients with renal failure: effect of various therapeutic approaches. East Afr Med J 74:503-9

36. Chothia MY, Halperin ML, Rensburg MA, et al (2014) Bolus administration of intravenous glucose in the treatment of hyperkalemia: a randomized controlled trial. Nephron Physiol 126:1-8

37. Harel Z, Kamel KS (2016) Optimal dose and method of administration of intravenous insulin in the management of emergency hyperkalemia: a systematic review. PLoS One 11:e0154963

38. Wheeler DT, Schafers SJ, Horwedel TA, et al (2016) Weightbased insulin dosing for acute hyperkalemia results in less hypoglycemia. J Hosp Med 11:355-7

39. McNicholas BA, Pham MH, Carli K, et al (2018) Treatment of hyperkalemia with a low-dose insulin protocol is effective and results in reduced hypoglycemia. Kidney Int Rep 3:328-36

40. Schafers S, Naunheim R, Vijayan A, et al (2012) Incidence of hypoglycemia following insulin-based acute stabilization of hyperkalemia treatment. J Hosp Med 7:239-42

41. Sterns RH, Grieff M, Bernstein PL (2016) Treatment of hyperkalemia: something old, something new. Kidney Int 89:546-54

42. Abuelo JG (2018) Treatment of severe hyperkalemia: confronting 4 fallacies. Kidney Int Rep 3:47-55

43. Sterns RH, Rojas M, Bernstein P, et al (2010) Ion-exchange resins for the treatment of hyperkalemia: are they safe and effective? J Am Soc Nephrol 21:733-5

44. Batterink J, Lin J, Au-Yeung SHM, et al (2015) Effectiveness of sodium polystyrene sulfonate for short-term treatment of hyperkalemia. Can J Hosp Pharm 68:296-303

45. Rafique Z, Kosiborod M, Clark CL, et al (2017) Study design of Real World Evidence for Treatment of Hyperkalemia in the Emergency Department (REVEAL-ED): a multicenter, prospective, observational study. Clin Exp Emerg Med 4:154-9

46. Pitt B, Bakris GL (2015) New potassium binders for the treatment of hyperkalemia: current data and opportunities for the future. Hypertension 66:731-8 